\title{
Harmless screening of humans for the detection of concealed objects
}

\author{
M. Kowalski, M. Kastek, M. Piszczek, M. Życzkowski \\ \& M. Szustakowski \\ Military University of Technology, Institute of Optoelectronics, Poland
}

\begin{abstract}
Multispectral screening systems are becoming more popular because of a wide range of applications. Terahertz and infrared radiation have unique properties applicable to the field of surveillance and security systems. One of the most significant applications of multispectral screening systems is the prevention of terrorist attacks. Visual detection of objects hidden under clothing of a person is one of the most challenging problems of threat detection. There are many solutions to the problem; however, the most effective utilize multispectral surveillance imagers. We investigate the possibility of harmlessly detecting objects covered by various types of clothing in three spectrums - visible, infrared and terahertz. The terahertz range of electromagnetic radiation has considerable potential to detect hidden objects because it penetrates clothes. The infrared imagers are also very useful in searching for concealed objects because infrared cameras can detect small temperature differences on the surface of clothing. Radiation from these three ranges is harmless to humans. We present the detection methodology using infrared and terahertz imagers as complementary sensors to provide image data for a multispectral threat detection system. Possible ways of using the results are also presented.

Keywords: THz imaging, object detection, harmless screening.
\end{abstract}

\section{Introduction}

A need to build highly efficient security systems for the detection of items covered with fabrics is a very urgent topic for researchers. Because of increased concern over terrorist attacks on aircraft, sensitive security scanners have been developed to screen passengers more effectively. Some of these scanners use 
ionizing radiation like X-rays, which is a type of radiation that can cause cancer and other health problems $[1,2]$. In order to scan people effectively without harmful effects other imaging techniques might be employed. We consider two ranges of radiation as potentially useful for detection of hidden objects and unconditionally harmless to humans.

One of the solutions is a terahertz $(\mathrm{THz})$ imager [3, 4]. Practical, real-time imaging in the terahertz band is of great interest for the screening of people, particularly the detection of dangerous objects at standoff range [5-7]. The terahertz waves offer great possibilities in the field of concealed objects detection because can penetrate various materials and are not harmful to humans [8]. However, the main difficulty in the $\mathrm{THz}$ imagers is low spatial resolutions and low image quality [8].

Another possible solution is a high resolution infrared camera. Because of the fact that infrared cameras can detect the temperature differences on the surface of the object, it is justified to investigate the possibilities of applying these type of imagers for detection of hidden items [9]. Both infrared and terahertz imagers are not harmful to humans.

A range of scanners using non-ionising radiation are currently being developed and assessed for security screening purposes. There are two types of this technology. Active scanners emit waves to produce an image. Passive scanners detect natural radiation emanating from the person. We focus on using passive imagers operating in the room temperature.

\section{Methodology of imaging}

Due to the fact that the thermal camera measure the relative temperature of objects and because during the measurements an object is covered with material, it measures the relative temperature of the surface of covering material. A thermal camera can be used to detect an object covered with a fabric only in certain conditions. The main condition is a value of temperature difference between an object and a surface of the covering material. In order to detect the temperature difference, the thermal sensitivity of an infrared imager should be as low as possible $[9,10]$.

The radiation distribution detected by the passive terahertz imager is proportional to the relative temperature of a target and is directly related to the absolute amount of $\mathrm{THz}$ radiation emitted by a target.

In normal operating conditions, the hidden object is heated by a human body (because of direct contact between the object and the human body), therefore the value of temperature difference between a covering material and an object may decrease. This fact should be considered in order to evaluate the potential of an infrared and terahertz cameras to detect hidden object.

However, the main difficulty in the $\mathrm{THz}$ imaging systems is low image quality due to low sensitivity and a small number of pixels in detecting modules of cameras.

Considering the fact that even $\mathrm{THz}$ images with low pixel resolution still provide valuable information, it is justified to combine them with the high- 
resolution images from a visible camera $[11,12]$. Image fusion is a process of combining two or more images, or it can be defined as the process by which several images or parts of images (features) are combined into a single image. This process allows us to combine the most interesting elements of images from various spectral ranges into one image.

The theory for performing visual tasks using cameras is described by Johnson's criteria [13]. The criteria define the conditions of an imager for the detection, recognition and identification of an object. Because of the relatively small pixel and spatial resolutions of modern terahertz cameras, it was not possible to recognize the object for detection based on the terahertz image [11]. The investigation into new non-destructive methods for the recognition of detected objects led to the concept of the methodology of multispectral detection $[11,12]$. The methodology is presented in Fig. 1.

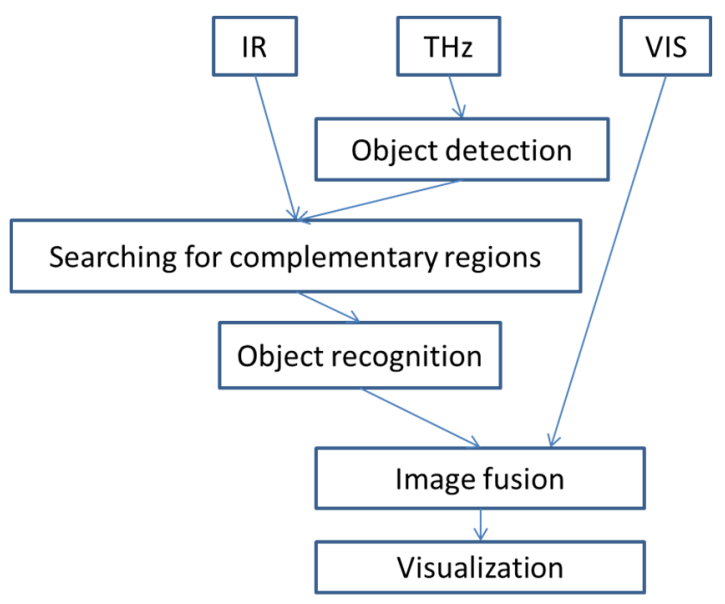

Figure 1: Methodology for detection and recognition of objects in multispectral systems.

\section{The experiment}

The experiment based on the detection methodology, presented in Fig. 1, employed three cameras operating in the visible, infrared and terahertz spectral ranges to detect, recognize and finally visualize the object. The methodology assumed that the terahertz camera was the primary sensor providing image data for the detection of objects, because of its proven capability of imaging through textiles. During the analysis of a terahertz image, regions with anomalies were considered as potential places to hide an object. The infrared image was searched to locate the complementary regions. The infrared image was exploited to achieve better quality imaging of an anomaly detected using the terahertz image. We investigated the potential to recognize the detected object by the analysis of the infrared image. In the last part of the methodology, the object - detected or 
identified - was extracted and fused with the visible image to provide the operator of the potential security system with clear and understandable visualization of the threat. The visible image operator enabled the person carrying the detected object to be identified.

In order to investigate the possibilities of detection of objects covered with various types of fabrics, the measurement methodology was developed. The measurement methodology consists of methods and algorithms used during the measurements as well as the hardware setup. The measurement setup consists of several devices - four cameras, two thermoelements (stuck to object and human body) and a thermo-higro-barometer. The cameras were selected to cover very wide range of spectra. The four cameras employed during the measurements visible light (VIS) camera, passive terahertz (THz) camera and two infrared cameras are commercially available. In order to provide a controllable and uniform background of measurement scene, a photographic fabric was used.

Complementary images acquired with the four cameras are presented in Fig. 2.

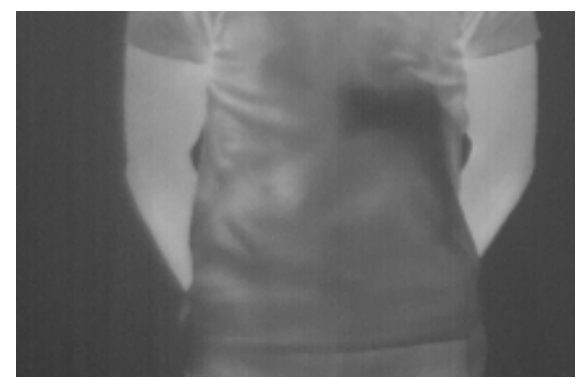

(a)

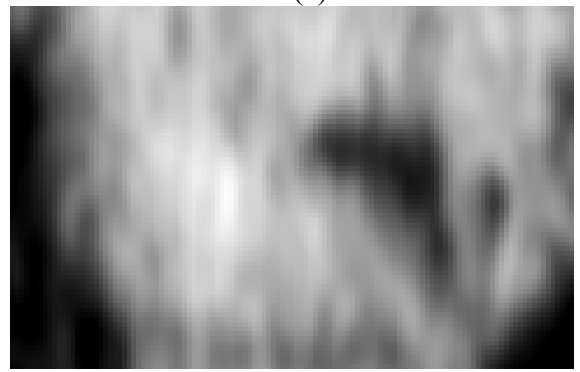

(c)

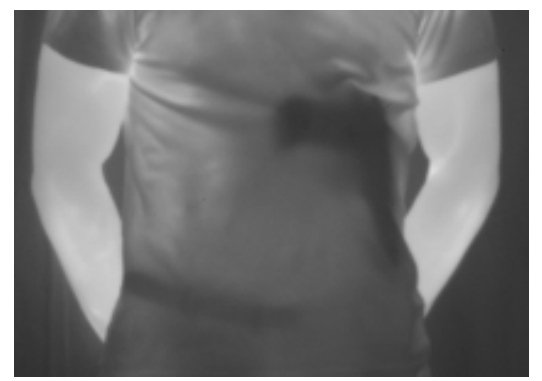

(b)

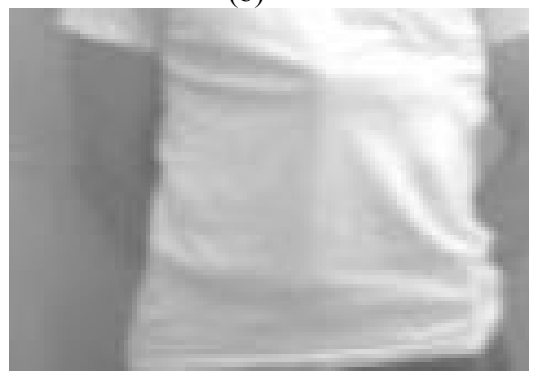

(d)

Figure 2: A human with concealed gun registered in (a) long-wavelength infrared (b) mid-wavelength infrared (c) terahertz and (d) visible range.

In order to investigate the possibilities of detection of hidden object in the terahertz range, the TS4 camera from ThruVision was employed. The cameras is operating at $250 \mathrm{GHz}$ and offers a resolution of 124x271 pixels [14]. The possibilities of detection of hidden objects in infrared range were investigated using two cameras. The first infrared camera used during the measurements is a 
long-wavelength infrared (LWIR) camera P640 from FLIR supplied with uncooled microbolometer detector working in the range of 7.5-13 $\mu \mathrm{m}$ and with a resolution of $640 \times 480$ pixels. The thermal sensitivity of the LWIR camera is $55 \mathrm{mK}$ at $30^{\circ} \mathrm{C}[15]$.

The second infrared camera is a mid-wavelength infrared (MWIR) SC5600 camera also manufactured by FLIR. The camera is supplied with cooled microbolometer detector $(3-5 \mu \mathrm{m})$ with resolution of $640 \times 480$ pixels. The thermal sensitivity of the MWIR camera is typically $20 \mathrm{mK}$ [15].

Organization of measurement sessions is presented in Fig. 2. During the measurements, several configurations with various objects (guns, knives, dynamite) and clothes (shirt, T-shirt, sweater) were prepared. A measurement of one single configuration took 30 minutes.

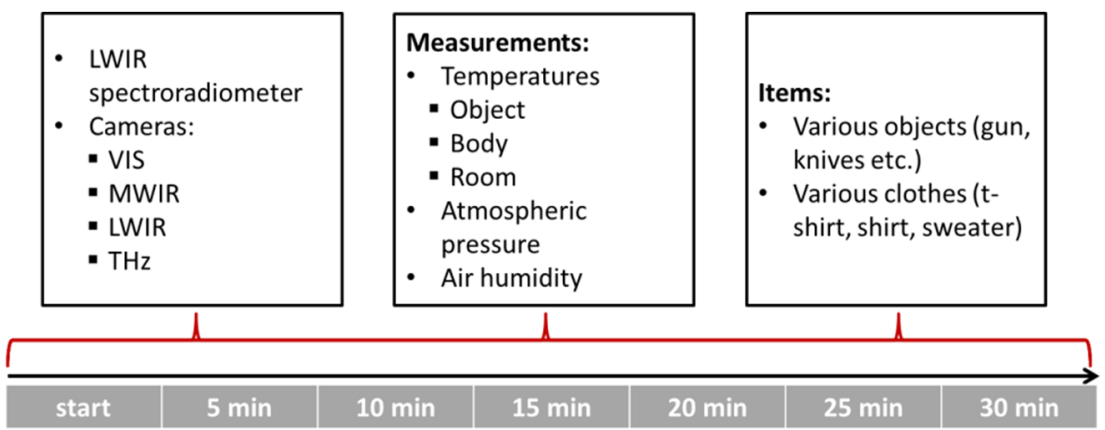

Figure 3: Measurement methodology.

The distance between a human and a set of measurement devices is $5 \mathrm{~m}$ and is constant during the measurements. A set of data from cameras (four images) and thermoelements were collected every five minutes. For every five minutes, the data package with images, values of atmosphere parameters (air temperature, humidity and pressure) and values of body and object temperatures was collected.

\section{Experimental results}

Selected images registered with four cameras (LWIR, MWIR, THz and VIS) and with test object (a gun) and one type of clothing (shirt) are presented in Figs 4, 5 and 6 respectively.

Images presented in Figs 4-6 were acquired at specified time intervals; at the beginning of the experiment, and after 15 and 30 minutes. Each experiment lasted 30 minutes. Experimental data from cameras were collected every 5 minutes. Before starting the experiments, the test objects and clothes were allowed to reach thermal equilibrium with the environment. 


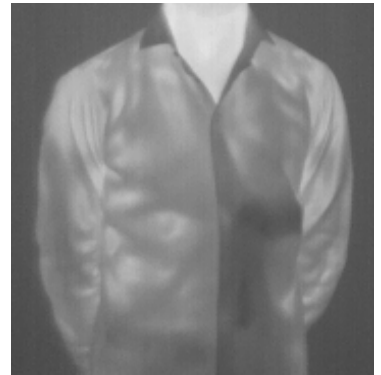

(a)

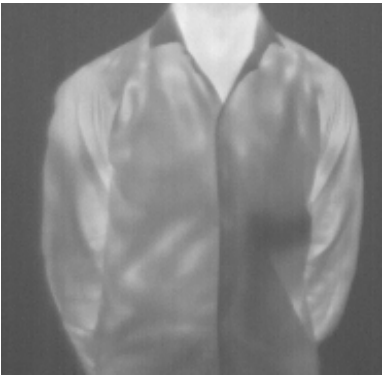

(b)

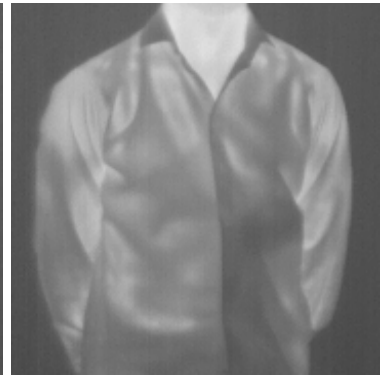

(c)

Figure 4: Images acquired by LWIR camera presenting a man wearing a shirt with a pistol, (a) at the beginning of the measurement session, (b) after 15 minutes, (c) after 30 minutes.

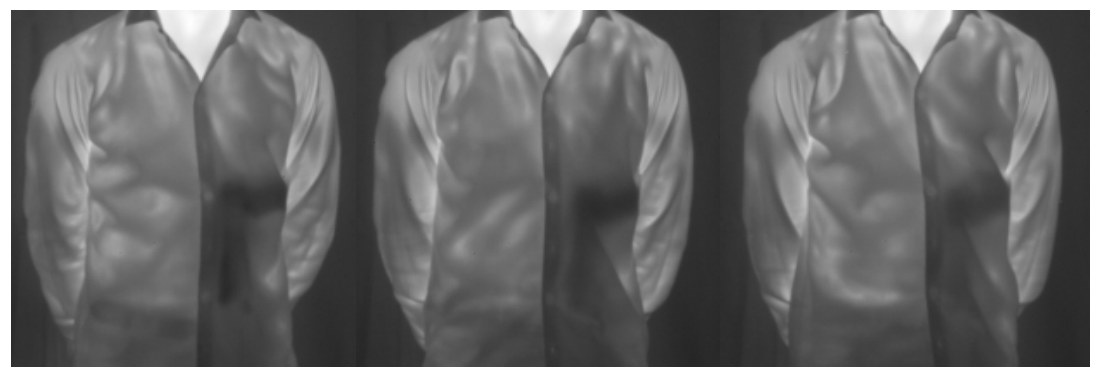

(a)

(b)

(c)

Figure 5: Images acquired by MWIR camera presenting a man wearing a shirt with a pistol, (a) at the beginning of the measurement session, (b) after 15 minutes, (c) after 30 minutes.

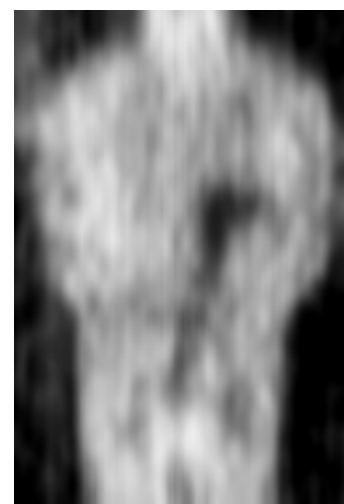

(a)

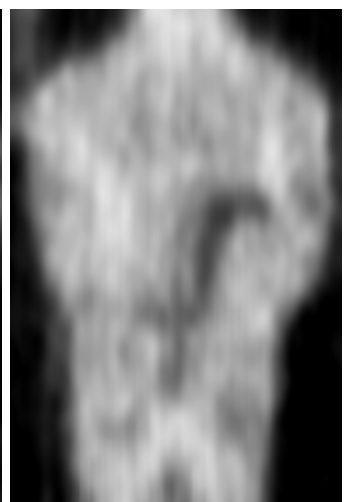

(b)

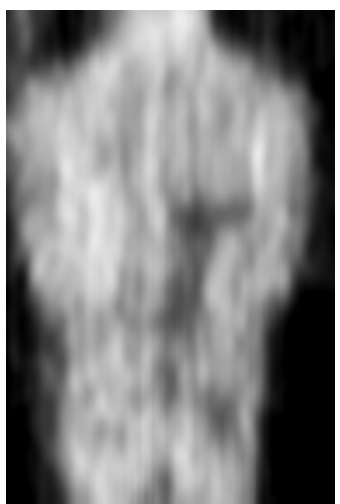

(c)

Figure 6: Images acquired by $\mathrm{THz}$ camera presenting a man wearing a shirt with a pistol, (a) at the beginning of the measurement session, (b) after 15 minutes, (c) after 30 minutes. 
According to the results presented in Figs 4-6, the contrast of the concealed object decreased during the experiment as a result of direct contact between the body and object. Because of the transfer of energy between the two, both endeavor to achieve thermal equilibrium. The temperature difference between the object and the body decreased. Therefore, the ability to detect the concealed object with the infrared and terahertz cameras depended on the temperature parameters of the observed objects and this became less effective during the experiment. Comparison of the images indicated that in the case of the terahertz images the contrast in temperature between the hidden object and the body was higher than in the case of LWIR images and did not decrease rapidly with time.

To present the numbers in comparable manner, pixel values have been normalized using the following equation:

$$
L(x, y)=\frac{I(x, y)}{\max (I)},
$$

where $L(x, y)$ is the value of a normalized pixel, $\max (I)$ is the maximum pixel value in the image, and $I(x, y)$ is the value of a pixel before normalization.

Subjectively, a location of the hidden object is visible in every image except the images acquired with visible light camera. It can be noticed that the visibility of the concealed object decreases during time. It is a result of decrease of temperature difference between the object and the human body. It should be noted that the ability to detect a concealed object with a camera depends on the type of clothes material and the thickness of clothing.

Comparing images registered with two thermal imagers it can be noticed that the hidden object is more distinct in the images registered with the MWIR camera.

However, to evaluate the capabilities of imager to detect a hidden object it is justified to process images in order to extract the hidden object. Image processing is an inevitable element of any surveillance security system. In the case of a system for detection of concealed objects, the system operator should be provided with a clear and understandable information [16]. The examples of binary images are presented in Fig. 7.

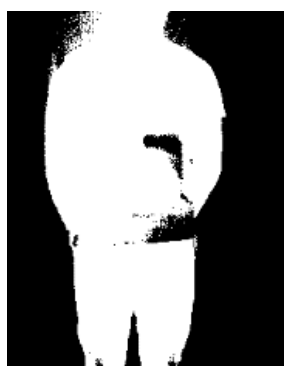

(a)

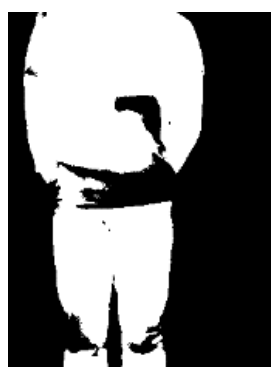

(b)

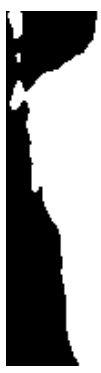

(c)

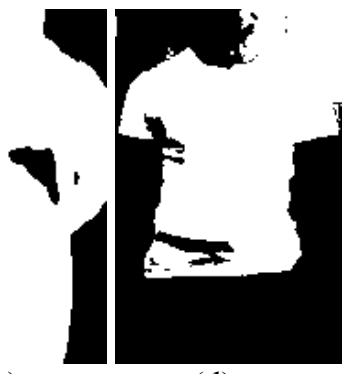

(d)

Figure 7: Processed images after binarization; (a) LWIR image, (b) MWIR image, (c) THz image, (d) VIS image. 
The processed images presented in Fig. 7 show the possibilities of extraction of detected object. The processed VIS image presented in Fig. 7(d) confirms that it is not possible to detect a concealed object with a single visible light camera. However, the VIS image can be utilized in the image fusion process [lit]. Processed thermal and terahertz images presented in Fig. 7(a)-(c) show the detected object. However, the image processing algorithms need to be improved because of the artifacts remaining in the images after processing.

\section{Conclusions}

The purpose of the studies was to investigate the possibilities of using various cameras operating in different spectral ranges for detection of concealed objects. In the article, we presented the measurement setup consisting of medium wavelength infrared (MWIR), long wavelength infrared (LWIR), THz and visible cameras and the initial results of measurements with various types of clothing and test objects. The basic idea of the measurements was to identify the possibility not only to detect the object covered with textiles but also to identify the object. The methodology of detection assumes that terahertz camera was the primary source of image and we investigated a possibility to use infrared image to identify the detected object by analysis of the infrared image.

The presented results indicate how the changes of temperature values of human body and the object can influence the camera's ability of detection of the object covered with fabrics. The results presented in the article confirm the fact that it is possible to detect a gun covered with a shirt placed on a human body using a thermal and terahertz imager in certain conditions.

The results indicate that during the measurements the intensity of pixels presenting the concealed object decreases due to the fact, that during the measurements, the hidden object is heated by a human body and the value of temperature difference between a covering material and an object decreases.

\section{References}

[1] Rez, P., Metzger, R. I., Mossman, K. L., The Dose From Compton Backscatter Screening, Oxford Journals: Radiation Protection Dosimetry, 45(1): pp. 75-81, 2011.

[2] Brenner, D.J., Are X-ray backscatter scanners safe for airport passenger screening? For most individuals, probably yes, but a billion scans per year raises long-term public health concerns, Radiology 259(1), pp. 6-10, 2011.

[3] Jansen, C., Wietzke, S., Peters, O., Scheller, M., Vieweg, N., Salhi, M., Krumbholz, N., Jördens, C., Hochrein, T., Koch, M., Terahertz imaging: applications and perspectives, Appl. Optics 49(19), pp. E48-E57, 2010.

[4] Kemp, M.C., Millimetre wave and terahertz technology for the detection of concealed threats: a review, Infrared and Millimeter Waves, pp. 647648, 2007. 
[5] Palka, N., Identification of concealed materials, including explosives, by terahertz reflection spectroscopy, Opt. Eng. 53(3), pp. 1-6, 2014.

[6] Lee, Y.S., Principles of Terahertz Science and Technology, Springer, New York, 2008.

[7] Zhang, X., Xu, J., Introduction to THz Wave Photonics, Springer, New York, 2010.

[8] Kowalski, M., Palka, N., Piszczek, M., Szustakowski, M., Hidden Object Detection System Based on Fusion of THz and VIS Images, Acta Phys. Pol. A 124(3), pp. 490-493, 2013.

[9] Kastek, M., Piątkowski, T., Dulski, R., Chamberland, M., Lagueux, P., Farley, V., Multispectral and hyperspectral measurements of soldier's camouflage equipment, Proc. SPIE 8382, p. 83820K, 2012.

[10] Kastek, M., Piątkowski, T., Trzaskawka, P., Infrared imaging Fourier transform spectrometer as the stand-off gas detection system, Metrology and Measurement Sys. 18(4), pp. 607-620, 2011.

[11] Kowalski, M., Kastek, M., Palka, N., Polakowski, H., Szustakowski, M., Piszczek, M., Investigation of concealed objects detection in visible, infrared and terahertz ranges of radiation, Phot. Lett. Poland 5(4), pp. 167$169,2013$.

[12] Kowalski, M., Kastek, M., Polakowski, H., Palka, N., Piszczek, M., Szustakowski, M., Multispectral concealed weapon detection in visible, infrared, and terahertz, Proc. SPIE 91020, pp. 91020T-91020T-7, 2014.

[13] Johnson, J., Analysis of image forming systems, Image Intensifier Symposium, pp. 244-273, 1958.

[14] ThruVision, www.digitalbarriers.com/products/thruvision/

[15] FLIR, www.flir.com/

[16] Kowalski, M., Palka, N., Piszczek, M., Szustakowski, M., Processing of $\mathrm{THz}$ images acquired by passive camera, Phot. Lett. Poland 4(3), pp. 9799, 2012. 